\title{
New Results on the Numerical Stability of the Stochastic Fluid Flow Model Analysis
}

\author{
Markus Fiedler ${ }^{1}$ and Holger Voos $^{2}$ \\ 1 University of Karlskrona/Ronneby \\ Dep. of Telecommunications and Signal Processing (ITS) \\ S-37179 Karlskrona, Sweden \\ phone: +46-708-537339, fax: +46-455-385667 markus.fiedler@its.hk-r.se \\ ${ }^{2}$ University of Kaiserslautern, Institute of Process Automation \\ PO-Box 3049, D-67653 Kaiserslautern, Germany \\ phone: +49-631-205 4457, fax: +49-631-205 4462, voos@eit.uni-kl.de
}

\begin{abstract}
The stochastic fluid flow model (SFF) is one of the leading models in performance evaluation for tele- and datacommunication systems, especially in fast packet-switching networks and ATM. However, the numerical analysis of the SFF is widely considered to be unstable. In this paper, some investigations and results are presented concerning the numerical stability of the SFF analysis also for large systems with finite buffer. We identify the main source of the numerical problems and give hints how to circumvent them. The usefulness of different solution methods are compared and the most robust methods for systems with large numbers of sources and large buffer sizes are identified.
\end{abstract}

\section{Introduction}

One possible model for performance evaluation in communications systems is the so called stochastic fluid flow model (SFF). It is widely used when dealing with fast packet-switched and ATM networks but also allows performance analysis in any kind of tele- and datacommunications system including mobile communications. First pioneering work considering the SFF can be found in [8]. In [1], an elegant treatment of a finite number of homogeneous on-off fluid sources whose streams were concentrated by a multiplexer with infinite buffer is presented. In 1984, Kosten presented an expansion of that model to heterogeneous traffic 9], and in 1988, Tucker published the formal way of how to treat finite multiplexer buffers 17. The main advantage of the SFF is the computational complexity which is independent of the buffer size [12.

However, the analysis of the SFF is widely considered to be numerically unstable. Indeed, the solution contains components that are exponentially increasing with the buffer content in the case of limited buffer size [8], [1], [17]. For that reason and the fact that no closed-form solutions exist, most work in the 1990's deals with buffers of infinite size or approximations of the solution based upon that assumption. In 1991, Nagarajan et al [13] reported results for 130 voice sources described in [7], which clearly differ from their simulation results. 
In 1995, Yang et al 18 described results for 25 homogeneous sources but were unable to obtain results for large-size problems due to numerical instability of their solution method. In spite of these difficulties, very few material exist that discusses these numerical problems in detail.

Therefore, this paper closes that gap between theory and numerical practice for the classical spectral method that is based on computing an eigensystem (spectral decomposition and on determining the coefficients associated to the spectral components. We are going to demonstrate how stable the spectral method actually might perform even for large systems with finite buffers if some quite simple rules are taken care of. In section 2, we describe the model and the notation that is used throughout this paper. Section 3 deals with the main steps of the stochastic fluid flow analysis. Section 4 introduces the numerical methods to solve the system of linear equations, which is necessary to adapt the solution of the system of differential equations to the boundary conditions. Furthermore, some special implementation issues are proposed. Section 5 presents numerical results and discusses the feasibility of different numerical methods for systems with homogeneous and heterogeneous sources. Section 6 summarizes the main results and gives an outlook on future work.

\section{The Stochastic Fluid Flow Model}

The SFF under investigation is the classical model which can be found in many publications. We assume $N$ on-off fluid sources that alternate randomly between an on state with peak cell rate $h$ and an off state, both of exponentially distributed duration. Thus, a discrete-valued rate process $S$ is formed with $n_{S}$ states $s_{i}$ and flow intensities $R \in\left\{r_{i}\right\}, i \in\left\{0, \ldots, n_{S}-1\right\}$. In addition, we assume a fluid buffer of finite size $K$ and an outlet (= server) with capacity $C$. The dynamic of the rate process $S$ is described by an irreducible Markov chain, represented by the infinitesimal generator matrix $\mathbf{M}$, which also determines the state probabilities $\pi_{i}$. Each state has a drift value $d_{i}=r_{i}-C$, depending on which the states are classified in

- over-load states $\mathcal{S}^{o}=\left\{i \mid d_{i}>0\right\}$;

- equilibrium states $\mathcal{S}^{e}=\left\{i \mid d_{i}=0\right\}$;

- under-load states $\mathcal{S}^{u}=\left\{i \mid d_{i}<0\right\}$.

These values are collected in the so called drift matrix $\mathbf{D}=\operatorname{diag}\left[d_{i}\right]$. The matrix $\mathbf{R}=\operatorname{diag}\left[r_{i}\right]$ is called rate matrix. We denote the aggregate state space with $\mathcal{S}=\mathcal{S}^{o} \cup \mathcal{S}^{e} \cup \mathcal{S}^{u}$. The $n$ on-off sources might be homogeneous, i. e. all have the same parameters, or heterogeneous. In the latter case, we form $n_{G}$ groups, each containing homogeneous sources.

\section{The Fluid Flow Analysis}

The following represents a summary of the fluid flow analysis which deals with the most essential points in the numerical context. Further information might be obtained from [1], [10], [15], [17]. 
Let $X$ be the buffer content of the fluid flow buffer with $0 \leq X<K$. Its stationary distribution function $\boldsymbol{F}(x)$ with elements $F_{i}(x)=\operatorname{Pr}\{X \leq x \wedge$ state $=$ $i$ \} is governed by the system of differential equations

$$
\mathbf{D} \cdot \frac{\mathrm{d}}{\mathrm{d} x} \boldsymbol{F}(x)=\mathbf{M} \cdot \boldsymbol{F}(x) \text {. }
$$

From (1), an eigenvalue-eigenvector problem

$$
z_{q} \mathbf{D} \cdot \varphi_{q}=\mathbf{M} \cdot \varphi_{q}
$$

with $q \in\left\{0, \ldots, n_{S}-1\right\}$ is obtained. The eigenvalues $z_{q}$ and eigenvectors $\boldsymbol{\varphi}_{q}$ appear in the so called spectral components in the solution of (1),

$$
\boldsymbol{F}(x)=\sum_{q \in \mathcal{S}} a_{q}(K) \boldsymbol{\varphi}_{q} \exp \left(z_{q} x\right) .
$$

For homogeneous on-off sources, eigenvalues and eigenvectors are given in closed form [1]. We normalize the eigenvectors in a way that the sum of all elements equals one. If the sources are heterogeneous, the set of eigenvalues $\left\{z_{q}\right\}$ has to be determined numerically from the inverse eigenvalue problem

$$
\gamma_{q}\left(z_{q}\right) \boldsymbol{\varphi}_{q}=\left(\mathbf{R}-\frac{1}{z_{q}} \mathbf{M}\right) \boldsymbol{\varphi}_{q} \quad \text { with } \quad \gamma_{q}\left(z_{q}\right)=\sum_{j=1}^{n_{G}} \gamma_{q}^{(j)}\left(z_{q}\right)=C
$$

For groups of on-off sources, the functions $\gamma_{q}^{(j)}\left(z_{q}\right)$ are also given in closed form and depend on the number of sources that are on in state $q$ and belong to group $j$. Once $z_{q}$ is determined, the corresponding eigenvector is a composition of parts that are determined for homogeneous groups. This composition is done using Kronecker algebra. More details might be found in [15], [10].

The coefficients $a_{q}(K)$ in (3) are necessary to adjust the solution to the boundary conditions, which depend on the buffer size $K$ :

$$
F_{i}(K)=\pi_{i}, i \in \mathcal{S}^{u} ; \quad F_{i}(0)=0, i \in \mathcal{S}^{o} .
$$

Insertion of (5) into (3) leads to a system of linear equations that has to be solved in order to obtain the coefficients:

$$
\begin{aligned}
& \sum_{q \in \mathcal{S}^{u} \cup \mathcal{S}^{o}} a_{q}(K) \varphi_{q i} \exp \left(z_{q} K\right)=\pi_{i} ; \quad i \in \mathcal{S}^{u} \\
& \sum_{q \in \mathcal{S}^{u} \cup \mathcal{S}^{o}} a_{q}(K) \varphi_{q i}=0 ; \quad i \in \mathcal{S}^{o}
\end{aligned}
$$

Originally, cases $d_{i}=0$ had to be excluded by choosing an appropriate value of $C$ [1]. However, we are able to deal with those equilibrium states in the same formal way as with over- or under-load states. Numerical investigations show 
that if the drift in a non-equilibrium state becomes arbitrarily small, the corresponding coefficient also escapes in the limit while the corresponding eigenvector approaches a unity vector in $q$-direction:

$$
\lim _{d_{q} \rightarrow 0} a_{q}(K)=0, \quad \lim _{d_{q} \rightarrow 0} \boldsymbol{\varphi}_{q}=\boldsymbol{e}_{q}
$$

This means that in the limit $d_{q} \rightarrow 0$, there is no coupling between equation $q$ and the other equations. Thus, we are free to ignore states with vanishing drift during the solution procedure.

\subsection{Finite Buffer Case}

The mostly ill-conditioned system of linear equations (6) of size ( $\operatorname{dim} \mathcal{S}^{u}+$ $\left.\operatorname{dim} \mathcal{S}^{o}\right) \leq n_{S}$ has to be solved numerically. Different methods to do this will be presented and evaluated in sections 4 and 5 . As soon as the coefficients are determined, the probability that the buffer is full in state $i$ is found to be

$$
u_{i}(K)=\pi_{i}-\lim _{b \rightarrow K} F_{i}(b)=\pi_{i}-\sum_{q \in \mathcal{S}} a_{q}(K) \boldsymbol{\varphi}_{q} \exp \left(z_{q} K\right),
$$

see [17]. With this, the loss probability can be expressed as

$$
P_{\mathrm{L}}(K)=\frac{\sum_{i \in \mathcal{S}^{\circ}} u_{i}(K) d_{i}}{\mathbf{E}[R]}
$$

\subsection{Buffer-Less Fluid Flow Model}

The so called buffer-less fluid flow model is obtained by passing the buffer size to the limit $K \rightarrow 0$. This case may be assumed if the buffer is much smaller than the mean burst length and thus (almost) looses its influence on burst level. In this case, the fluid flow analysis described before doesn't need to be carried out anymore which allows the computation of systems with very large state spaces. Loss happens as soon as positive drift occurs, so that the probabilities $u_{i}$ in the loss probability formula (9) can be replaced by the corresponding state probabilities $\pi_{i}$ :

$$
P_{\mathrm{L}}(0)=\frac{\sum_{i \in \mathcal{S}^{\circ}} \pi_{i} d_{i}}{\mathbf{E}[R]}
$$

\section{Numerical Methods}

In this section we present a selection of methods that have been used to solve the system of linear equations (6) which is mostly ill-conditioned, not sparse, not symmetrical and not positive definite. In addition, the systems are large (depending on the size of the state space $\mathcal{S}$ ) and associated with numerical input errors that stem from the numerical determination of the eigensystem. Our aim is to check how the following methods work in spite of these difficulties. Since the classification of the methods is not unique in the literature, we refer to the classification given by Stewart [16]. 


\subsection{Direct Methods}

With direct methods, we describe "numerical methods that compute solutions of mathematical problems in a fixed number of operations" [16]. We focus on methods that transform a system of linear equations $\mathbf{A} \cdot \boldsymbol{x}=\boldsymbol{b}$ into a system $\mathbf{A}^{\prime} \cdot \boldsymbol{x}=\boldsymbol{b}^{\prime}$ during a so-called reduction phase. Herein, $\mathbf{A}^{\prime}$ represents an upper triangular matrix. Then, the components of the solution vector $\boldsymbol{x}$ are found by back substitution. See [5], 16], 4] for details on the following methods:

1. Gaussian elimination. The reduction is achieved by a special succession of divisions and subtractions of rows. Assume that in step $i$ the first $i-1$ rows have already been treated. The elements of row $i$ are obtained by

$$
a_{k l}^{(i)}=a_{k l}^{(i-1)}-\frac{a_{k i}^{(i-1)}}{a_{i i}^{(i-1)}} a_{i l}^{(i-1)} \quad k>i, l=1 \ldots n .
$$

The elements $a_{i i}^{(i-1)}$ are called pivots.

2. Gaussian elimination with partial pivoting. From a numerical point of view, the elements on the diagonal might not represent optimal pivots. It can be shown that the absolute value of the pivot should be as large as possible. Hence, if a pivot with larger absolute value can be obtained from another row in the same column, then the corresponding rows are interchanged.

3. Gaussian elimination with full pivoting. Here, an optimal pivot is searched in rows and columns. However, interchanging of columns leads to interchanged elements in the solution vector (a system $\mathbf{A}^{\prime} \cdot \boldsymbol{x}^{\prime}=\boldsymbol{b}^{\prime}$ appears), which have to be re-changed after back substitution.

4. Givens rotations. The reduction is performed by multiplying the system of equations with elementary rotation matrices, hereby cancelling out the elements in the lower triangular part. In [16], this method can be found among projection methods.

5. Householder transformation. This method also uses transformation matrices for the reduction phase. For details, see [1].

The methods 3 to 5 are stable with regard to the algorithm error.

\subsection{Iteration Methods}

Iteration methods try to approximate the solution by carrying out iterations, consisting of a couple of operations, as long as the approximation $\boldsymbol{x}^{*}$ has not yet converged to a desired extent. Starting from $\boldsymbol{x}^{*(0)}=\mathbf{0}$, the result of an iteration serves as basis for the next iteration, i. e. $\boldsymbol{x}^{*(i+1)}=f\left(\boldsymbol{x}^{*(i)}\right)$. To get a positive definite matrix instead of $\mathbf{A}$ that guarantees convergence [5], we first apply a so called Gaussian transformation by multiplying our system of equations with the transposed matrix $\mathbf{A}^{T}$ from the left side, i.e. we treat the system $\mathbf{A}^{T} \cdot \mathbf{A} \cdot \boldsymbol{x}=\mathbf{A}^{T} \cdot \boldsymbol{b}$. See again [5], [16, 4] for details on the following methods, which we use in a more direct way, as we fix the (maximal) number of iterations to 1000 : 
1. Gauss-Seidel iteration. The iteration formula reads

$$
x_{i}^{*(k+1)}=x_{i}^{*(k)}+\frac{\varepsilon}{a_{i i}} \cdot\left(b_{i}-\sum_{j=1}^{i-1} a_{i j} x_{j}^{*(k+1)}-\sum_{j=i}^{n} a_{i j} x_{j}^{*(k)}\right)
$$

with $\varepsilon=1$.

2. Incomplete relaxation. The speed of the convergence of the Gauss-Seidel method, which tries to minimize (= relax) the defect vector $\boldsymbol{b}-\mathbf{A} \cdot \boldsymbol{x}^{*}$ completely, might be improved by over-relaxation with $\varepsilon>1$. However, the relaxation factor $\varepsilon$ has to be chosen carefully; it depends heavily on the system of equations under study.

3. Jacobi rotation. This method uses the same kind of rotation matrices as Givens rotations to "improve" the matrix $\mathbf{A}^{T} \cdot \mathbf{A}$ by making it diagonally dominant. The grade of this dominance is specified by the rotation limit and the rotation stops as soon as the desired grade is reached. In this work, the algorithm stops in any case after 1000 iterations. Finally, a direct method delivers the solution.

All these methods are sensitive to rounding errors.

\subsection{Implementation Issues}

A proper numerical evaluation of the eigensystem has to be ensured in order to keep the input error for the solution of the system of linear equations (6) as small as possible. Therefore, the eigenvalues should be obtained with great precision which is no problem for on-off sources, even if a numerical search based on (4) was performed. But also the eigenvectors $\varphi_{q}$ should be determined with care even if the orders of magnitude of certain elements are extremely small. Section 5.3 demonstrates what happens if the precision of the eigenvector calculation drops. Note that due to $\lim _{d_{i} \rightarrow \pm 0} z_{i}=\mp \infty$, states with small drifts might cause numerical under-/overflow in (3). But (7) shows that the contribution of such components becomes arbitrarily small. Hence, they can be extracted before solving the system of linear equations (6). As floating-point variables with double precision range from about $\pm 10^{-323}$ to about $\pm 10^{308}$, we deleted such equations whose presence would probably lead to overflow in column $q$ due to $\exp \left(z_{q} K\right)>10^{300}$ or to zeros due to $\exp \left(z_{q} K\right)<10^{-300}$. After this reduction, a number of $n_{S}^{\prime}$ equations is left.

As public and commercial tools for numerical mathematics mostly do not allow the user to look inside their implementation, we wrote own code for the solution methods in $\mathrm{C}++$ with great care to avoid bad numerical surprises. To avoid excessive memory consumption and executing times (c.f. section [5.3), floating-point variables with double precision (8 Bytes) were used instead of such ones with long double precision (16 Bytes). 


\section{$5 \quad$ Results}

In this section, we present the quality of numerical results for loss probabilities in quite large systems with homogeneous and heterogeneous sources, which have been obtained on computers of the types Sun SparcStation 10 and 20 (architecture sun4m). Results that were obviously wrong, e.g. negative values, have been marked with "_.".

In relation to the mean burst sizes of the on-off sources under consideration, buffer sizes are classified as follows:

1. XS: Very small buffer that has no influence on burst level.

2. S: Small buffer of 0.1 times the minimal mean burst size.

3. M: Medium-sized buffer of the minimal mean burst size.

4. L: Large buffer of 10 times the minimal mean burst size.

5. XL: Very large buffer of 100 times the minimal mean burst size.

In case 1 , numerical results might be compared with very stable results provided by the buffer-less fluid flow model. Therefore, results delivered by the fluid flow analysis are regarded to be good enough if the corresponding relative error does not surpass $0.1 \%$. However, in the cases 2 to 5 , fluid flow simulations had to be used in order to obtain reference values. Here, the error tolerance was set to the $95 \%$ confidence interval, whose size was mostly less than $4 \%$ of the corresponding average value. A method for solving the system of linear equations (6) is considered as applicable for one of the following systems if the corresponding error tolerance is kept.

\subsection{Homogeneous System}

The homogeneous system under study consists of $N \in\{50,100, \ldots, 600\}$ quite bursty on-off sources with a mean-to-peak bit rate ratio of 0.1 . These sources are much more bursty and thus more critical than the voice model in [7]. The capacity of the multiplexer was chosen in a way that the load equals 0.8 ; the size of the state space is $n_{S}=N+1$.

XS buffer: Table 1 compares results obtained by using one direct and two iterative methods with reference results obtained from the buffer-less analysis. The

Table 1. Loss probabilities obtained with some solution methods, given homogeneous sources and a very small buffer.

\begin{tabular}{|r||c|c|c|c|}
\hline$N$ & Full pivot. & Gauss-Seidel & Incompl. relax. (factor) & Jacobi/Buffer-less \\
\hline \hline 100 & $3.7389 \times 10^{-2}$ & $3.6235 \times 10^{-2}$ & $3.6955 \times 10^{-2}(1.9)$ & $3.7389 \times 10^{-2}$ \\
200 & $1.3768 \times 10^{-2}$ & $1.3532 \times 10^{-2}$ & $1.3711 \times 10^{-2}(1.9)$ & $1.3768 \times 10^{-2}$ \\
300 & $6.6872 \times 10^{-3}$ & $6.6069 \times 10^{-3}$ & $6.6875 \times 10^{-3}(1.9)$ & $6.6872 \times 10^{-3}$ \\
400 & $3.4852 \times 10^{-3}$ & $3.4590 \times 10^{-3}$ & $3.4851 \times 10^{-3}(1.4)$ & $3.4852 \times 10^{-3}$ \\
500 & $1.9875 \times 10^{-3}$ & $1.9799 \times 10^{-3}$ & $1.9894 \times 10^{-3}(1.2)$ & $1.9875 \times 10^{-3}$ \\
600 & - & $1.1475 \times 10^{-3}$ & $1.1475 \times 10^{-3}(1.0)$ & $1.1480 \times 10^{-3}$ \\
\hline
\end{tabular}


Table 2. Usefulness of the solution methods given homogeneous sources and a very small buffer.

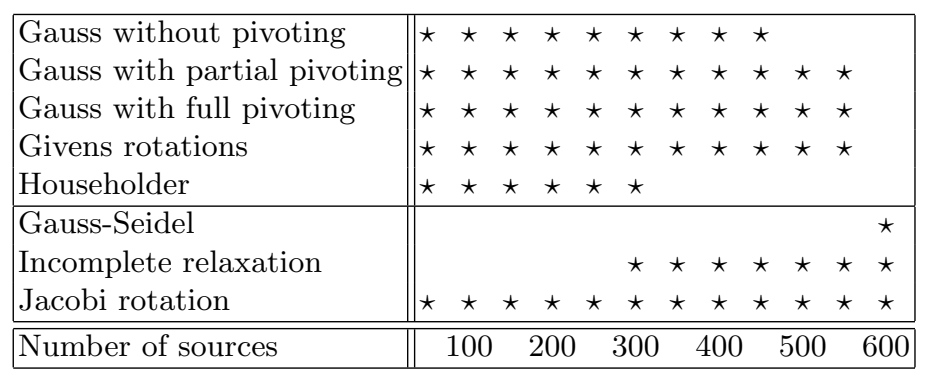

Gaussian elimination with full pivoting, which behaves best among the direct methods, reproduces the reference values up to 550 sources, but then fails. The Householder method already fails if the number of sources exceeds 300 . The Gauss-Seidel method produces better results the more the size of the system increases. The incomplete relaxation method leads to further improvement, but only if the relaxation factor has been optimized before. As there are reference values available, we chose the best result for each $N$ based on relaxation factors $\in\{1.0,1.1, \ldots, 1.9\}$. The Jacobi method with a rotation limit of 0.9 reproduced the reference values exactly for any number of sources, no matter which direct method is used at its end. For a number of 600 sources, only the iteration methods are still applicable. Table 2 summarizes the cases in which the different methods produced results within the defined error tolerance of $0.1 \%$; these cases are marked by " $\star$ ".

M buffer: Table 3 shows some results obtained with direct methods and compares them with simulation results.

For 550 sources, the Gaussian elimination without pivoting deviates obviously, while Givens rotations is the only method that manages 600 sources with acceptable precision. As in the previous case, the Householder method is not able to treat more than 300 sources. Among the iterative methods, most of the Gauss-Seidel results (not shown) lie within the corresponding confidence interval. Due to the lack of reference values, i.e. the possibility to optimize the relaxation factor, the incomplete relaxation method is not taken into account. No matter which direct method is used afterwards, the Jacobi rotation does not allow to

Table 3. Loss probabilities obtained with some solution methods given homogeneous sources and a medium-sized buffer.

\begin{tabular}{|r||c|c|c||c|}
\hline$N$ & Gauss & Full pivot. & Givens rot. & Simulation \\
\hline \hline 300 & $3.5543 \times 10^{-3}$ & $3.5544 \times 10^{-3}$ & $3.5544 \times 10^{-3}$ & $(3.5701 \pm 0.1202) \times 10^{-3}$ \\
350 & $2.5045 \times 10^{-3}$ & $2.5044 \times 10^{-3}$ & $2.5044 \times 10^{-3}$ & $(2.4981 \pm 0.0864) \times 10^{-3}$ \\
400 & $1.7992 \times 10^{-3}$ & $1.7980 \times 10^{-3}$ & $1.7980 \times 10^{-3}$ & $(1.7819 \pm 0.0553) \times 10^{-3}$ \\
450 & $1.3130 \times 10^{-3}$ & $1.3133 \times 10^{-3}$ & $1.3134 \times 10^{-3}$ & $(1.3034 \pm 0.0550) \times 10^{-3}$ \\
500 & $9.6838 \times 10^{-4}$ & $9.7026 \times 10^{-4}$ & $9.7302 \times 10^{-4}$ & $(9.7176 \pm 0.3259) \times 10^{-4}$ \\
550 & $7.6164 \times 10^{-4}$ & $7.2428 \times 10^{-4}$ & $7.2230 \times 10^{-4}$ & $(7.0691 \pm 0.2103) \times 10^{-4}$ \\
600 & - & - & $5.4560 \times 10^{-4}$ & $(5.2717 \pm 0.2138) \times 10^{-4}$ \\
\hline
\end{tabular}


Table 4. Usefulness of the solution methods given homogeneous sources and a mediumsized buffer.

\begin{tabular}{|c|c|c|c|c|c|c|}
\hline Gauss without pivoting & $\star \star$ & $\star \star$ & $\star$ & $\star \star$ & $\star$ & \\
\hline Gauss with partial pivoting & $\star \star \star$ & $\star$ & $\star$ & $\star$ & 夫 & $\star$ \\
\hline Gauss with full pivoting & $\star \star \star$ & $\star$ & $\star$ & $\star$ & $\star$ & $\star$ \\
\hline Givens rotations & 夫 & $\star$ & $\star$ & $\star$ & $\star$ & $\star$ \\
\hline Householder & $\star$ & $\star \star$ & $\star$ & & & \\
\hline Gauss-Seidel & 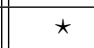 & $\star \star$ & $\star$ & $\star$ & $\star$ & \\
\hline Jacobi rotation & $\star$ & $\star \star$ & $\star \star$ & $\star \star$ & & \\
\hline Number of sources & 100 & 200 & 300 & 400 & 500 & 600 \\
\hline
\end{tabular}

Table 5. Usefulness of the solution methods given homogeneous sources and a large buffer.

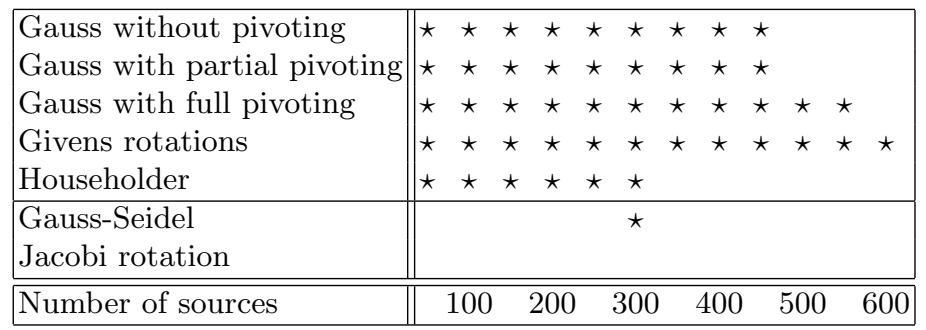

treat more than 400 sources. The results are summarized in Table 4 , where " $\star$ " stands for a result that lies within the corresponding confidence interval.

L buffer: For L buffer, the behavior of the direct methods is quite similar to that in the M buffer case. We confine ourselves to the summary that is presented in Table 5. The Gauss-Seidel method shows larger deviations as in the M buffer case; merely the result for 300 sources lies within the corresponding confidence interval, while the method fails for more than 350 sources. The Jacobi rotation was not able to produce any meaningful result. For L buffer, the direct methods seem to be much better applicable than the considered iteration methods.

\subsection{Heterogeneous System}

Here, we assume two groups of 30 on-off sources, each with the same mean bit rate and different parameters as follows:

1. Mean-to-peak bit rate ratio of 0.5 (less bursty), ratio of buffer and mean burst size $K / h b_{1} \in\{0 ; 1,10,100\}$;

2. Mean-to-peak bit rate ratio of 0.1 (more bursty), ratio of buffer and mean burst size $K / h b_{2} \in\{0 ; 1,10,100\}$.

For XS buffer, each direct method is able to compute the corresponding reference value, but table 6 reveals that the Gauss-Seidel method delivers results dependent on the burst sizes, which does not hold for the underlying bufferless model. This behavior might be interpreted as a signal for the Gauss-Seidel method's sensitivity to input errors. 
Table 7 shows results for the Gaussian methods for different buffer sizes. The result for XL buffer is too small to be compared with simulation results. A comparison with the upper bound for the loss probability that is based on the dominant eigenvalue [6], 2] excludes the results obtained with the Gaussian method without and with partial pivoting; the same is valid for Givens rotations results that are not shown explicitly. As this upper bound is known to overestimate the real result by several orders of magnitude, the result obtained with the Gaussian method with full pivoting seems reasonable. This method seems to be best among the direct methods when dealing with small probabilities. On the other hand, Householder and Gauss-Seidel fail in cases of large buffer sizes. Table 8 shows a summary.

\subsection{The Role of the Eigenvectors}

In this section, we show to which extent the accuracy of the eigenvectors directly influences the numerical stability of the solution methods. Here, only the homogeneous systems need to be investigated (in the heterogeneous case, the eigenvectors are obtained as a composition of parts that are determined for homogeneous groups). Since in this homogeneous case closed formula descriptions of the eigenvalues exist, they can be computed with great precision. However, during the calculation of the eigenvectors, huge differences in the orders of magnitude occur: there are products that contain terms causing numerical overflow as well as numerical underflow while the resulting product would lie in the tractable range concerning the order of magnitude. In our system, the use of floating-point numbers with double precision (8 Bytes) within that critical product restricted the number of sources to 100 .

A first improvement was reached by a separate treatment of base and exponent of eigenvector elements in two floating-point numbers with double precision, i.e. a kind of logarithmic calculation within the critical product. Using this trick, the number of sources could be raised to 250. The price to be paid: On average, the calculation of the eigenvectors took more than 10 times as long. However, the results for up to 600 sources that were reported in the previous section have been obtained using long double floating-point numbers (16 Byte). Compared to the use of the standard double data type, the speed went down by a factor of more than 100. In general, the effect of input errors on numerical stability seem to grow as the buffer becomes larger. Due to (3), this is not surprising: The eigenvectors are multiplied by exponential functions containing the

Table 6. Loss probabilities obtained with the Gauss-Seidel method for heterogeneous sources with different mean burst sizes and very small buffer.

\begin{tabular}{|r|r||c||c|}
\hline$h b_{1}$ & $h b_{2}$ & Gauss-Seidel & Reference \\
\hline \hline 10 & 10 & $2.4288 \times 10^{-3}$ & $2.4376 \times 10^{-3}$ \\
10 & 100 & $2.4260 \times 10^{-3}$ & $2.4376 \times 10^{-3}$ \\
100 & 10 & $2.4489 \times 10^{-3}$ & $2.4376 \times 10^{-3}$ \\
100 & 100 & $2.4289 \times 10^{-3}$ & $2.4376 \times 10^{-3}$ \\
\hline
\end{tabular}


Table 7. Loss probabilities obtained with Gaussian elimination methods for heterogeneous sources and M to XL buffers.

\begin{tabular}{|r|r||c|c|c|c|}
\hline$\frac{K}{h b_{1}}$ & $\frac{K}{h b_{2}}$ & No pivoting & Partial pivot. & Full pivot. & Sim./Approx. \\
\hline \hline 1 & 1 & $4.2648 \times 10^{-4}$ & $4.2648 \times 10^{-4}$ & $4.2648 \times 10^{-4}$ & $(4.2132 \pm 0.1059) \times 10^{-4}$ \\
10 & 10 & $1.1415 \times 10^{-7}$ & $1.1415 \times 10^{-7}$ & $1.1415 \times 10^{-7}$ & $(1.1240 \pm 0.0819) \times 10^{-7}$ \\
100 & 100 & $1.0778 \times 10^{-18}$ & $7.5006 \times 10^{-19}$ & $1.5120 \times 10^{-37}$ & Approx.: $1.2992 \times 10^{-33}$ \\
\hline
\end{tabular}

Table 8. Usefulness of the solution methods for different buffer sizes given heterogeneous sources.

\begin{tabular}{|l||cccc|}
\hline Gauss without pivoting & $\star$ & $\star$ & $\star$ & \\
Gauss with partial pivoting & $\star$ & $\star$ & $\star$ & \\
Gauss with full pivoting & $\star$ & $\star$ & $\star$ & $\star$ \\
Givens rotations & $\star$ & $\star$ & $\star$ & \\
Householder & $\star$ & $\star$ & & \\
\hline Gauss-Seidel & $\star$ & $\star$ & & \\
\hline \hline Buffer size: & XS & M & L & XL \\
\hline
\end{tabular}

buffer size. Especially iteration methods seem to suffer from this problem; even the powerful Jacobi rotation method fails. The Gaussian elimination with full pivoting seems to be the best possible method to get along with this problem.

\section{Conclusions}

In this paper, we presented some recipes on how to stabilize the stochastic fluid flow analysis numerically if finite buffers and the classical spectral method are used. The most essential step is to determine the eigensystem as precisely as possible. This should be done to keep the input error for the next step low, which is the solution of a system of linear equations in order to adapt the solution of the system of differential equations to the boundary conditions. Before solving that system, those equations that belong to states with very small drift values should be extracted. These states have no significant influence on the solution but may cause numerical under- or overflow. To solve the system of linear equations, the solution method has to be chosen very carefully. Considering the list of possible solution methods that were tested in this work, the use of the Gaussian algorithm with complete pivoting led to the best results, followed by the Givens rotations method. Although the summary tables presented in section 5 are valid only for the corresponding parameter settings, they reflect the experience we also made with other systems. Altogether, the proposed measures lead to a numerical performance of the stochastic fluid flow model analysis that is much better than its reputation. Future work on mumerical problems in the fluid flow model context should also consider the matrix-analytic solution by Ramaswami [14] as well as more complicated source models. 


\section{References}

1. D. Anick, D. Mitra and M. M. Sondhi. Stochastic theory of a datahandling system with multiple sources. The Bell System Technical Journal, 61(8):1871-1894 (1982).

2. A.I. Elwalid and D. Mitra. Effective bandwitdh of general Markovian traffic sources and admission control of high speed networks. IEEE/ACM Transactions on Networking, 1(3):329-343 (1993).

3. A.I. Elwalid and D. Mitra. Statistical multiplexing with loss priorities in rate-based congestion control of high-speed networks. IEEE Transactions on Communications, 42(12):2989-3002 (1994).

4. Encyclopaedia of mathematics. Kluwer, 1995, ISBN 1-55608-010-7.

5. D. K. Faddeev and V. N. Faddeeva. Computational methods of linear algebra. Freeman, 1963.

6. R. Guérin, H. Ahmadi and M. Naghshineh. Equivalent capacity and its application to bandwidth allocation in high-speed networks. IEEE Journal on Selected Areas in Communications, 9(7):968-981 (1991).

7. H. Heffes and D.M. Lucantoni. A Markov modulated characterization of packetized voice and data traffic and related statistical multiplexer performance. IEEE Journal on Selected Areas in Communications, 4(6):856-867 (1986).

8. L. Kosten. Stochastic theory of a multi-entry buffer. Delft Progress Report, Series F, 1:10-18 (1974).

9. L. Kosten. Stochastic theory of data handling systems with groups of multiple sources. Proc. of IFIP WG 7.3 - TC 6 International Symposium on the Performance of Computer Communication Systems, Zürich, 1984, pp 321331.

10. K.P. Kontovasilis and N.M. Mitrou. Bursty traffic modelling and efficient analysis algorithms via fluid-flow models for ATM IBCN. Annals of Operations Research, 49:279-323 (1994).

11. P. Lancaster and M. Tismentzky. The Theory of Matrices, Second edition with Applications. New York: Academic Press, 1985.

12. D. Mitra. Stochastic theory of a fluid flow model of producers and consumers coupled by a buffer. Advances in Applied Probability, 20:646-676 (1988).

13. R. Nagarajan, J.F. Kurose and D. Towsley. Approximations techniques for computing packet loss in finite-buffered voice multiplexers. IEEE JSAC, 9(3):368-377 (1991).

14. V. Ramaswami. Matrix analytic methods for stochastic fluid flows. Proc. ITC-16, Edinburgh, 1999, pp 1019-1030.

15. T. Stern and A. Elwalid. Analysis of separable markov-modulated rate models for information-handling systems. Advances in Applied Probability, 23:105-139 (1991).

16. W.J. Stewart. Introduction to the Numerical Solution of Markov Chains. Princeton University Press, 1994, ISBN 0-691-03699-3.

17. R. Tucker. Accurate method for analysis of a packet-speech multiplexer with limited delay. IEEE Transactions on Communications, 36(4):479-483 (1988).

18. T. Yang and D.H.K. Tsang. A novel approach to estimating the cell loss probability in an ATM multiplexer loaded with homogeneous on-off sources. IEEE Transactions on Communications, 43(1):117-126 (1995). 